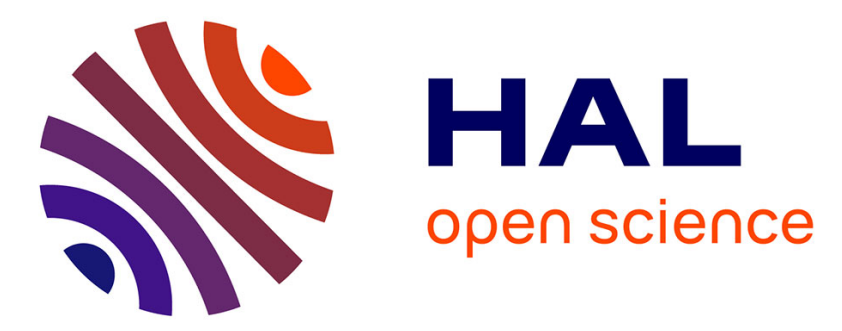

\title{
Sulforhodamine B-LDH composite as a rare-earth-free red-emitting phosphor for LED lighting
}

Paul Legentil, Fabrice Leroux, Sandrine Therias, Damien Boyer, Geneviève Chadeyron

\section{- To cite this version:}

Paul Legentil, Fabrice Leroux, Sandrine Therias, Damien Boyer, Geneviève Chadeyron. Sulforhodamine B-LDH composite as a rare-earth-free red-emitting phosphor for LED lighting. Journal of Materials Chemistry C, 2020, 8 (34), pp.11906-11915. 10.1039/D0TC02802A . hal-02992421

\section{HAL Id: hal-02992421 \\ https://hal.science/hal-02992421}

Submitted on 20 Nov 2020

HAL is a multi-disciplinary open access archive for the deposit and dissemination of scientific research documents, whether they are published or not. The documents may come from teaching and research institutions in France or abroad, or from public or private research centers.
L'archive ouverte pluridisciplinaire HAL, est destinée au dépôt et à la diffusion de documents scientifiques de niveau recherche, publiés ou non, émanant des établissements d'enseignement et de recherche français ou étrangers, des laboratoires publics ou privés. 


\title{
Sulforhodamine B-LDH composite as a rare-earth-free red-emitting phosphor for LED lighting.
}

\author{
Paul Legentil ${ }^{\mathrm{a}}$, Fabrice Leroux ${ }^{\mathrm{a} *}$, Sandrine Therias ${ }^{\mathrm{a}}$, Damien Boyer ${ }^{\mathrm{a}}$ and Geneviève Chadeyron ${ }^{\mathrm{a} *}$
}

Domestic lighting using white light-emitting diodes (WLEDs) requires a choice of materials to be eco-friendly as well as economically viable. Revisiting the optical properties of sulforhodamine B (SRB) with a new focus - designing rare-earth-free phosphors - is found here to be highly promising. Relying on a Layered Double Hydroxide (LDH) host, the dye molecules are stabilized, and the inorganic-organic assembly formed is dispersed into silicone. Homogeneous composite films present suitable and stable photometric parameters, making them appropriate candidates to replace red-emitting phosphors based on rare-earth elements.

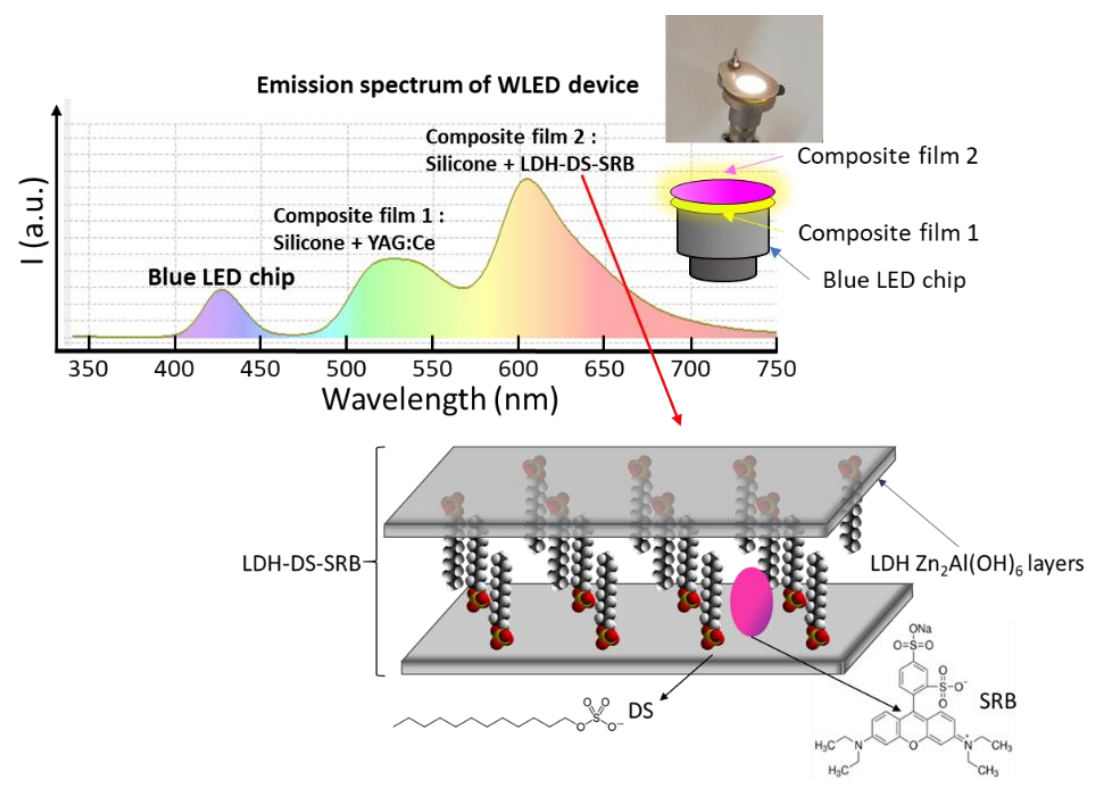

\section{Introduction}

White light-emitting diodes (WLEDs) are undoubtedly the new generation of solid-state lighting sources, because of their excellent features such as high luminous efficiency, energy saving properties, long lifetime and absence of toxic mercury. Currently, the most popular approach to producing WLEDs consists in the combination of a blue chip, emitting between 450 to $480 \mathrm{~nm}$, and YAG:Ce ${ }^{3+}$, a yellow phosphor. ${ }^{1}$ The main drawback to this system arises from its weak emission in the red spectral range, yielding a poor colour rendering index (CRI) and a cool colour temperature.

Therefore, a red-emitting phosphor is commonly added to this system to improve the colorimetric characteristics of the light. Numerous studies addressing the synthesis of red phosphors compatible with blue chips are reported in the literature. ${ }^{2}$ These red phosphors must fulfil several requirements, such as a good stability under photonic and thermal stresses, in addition to a high emission quantum yield. Sulfides and nitrides doped with $\mathrm{Eu}^{2+}$ are generally used as redemitting phosphors in commercial WLEDs, although they suffer from several drawbacks, including poor thermal stability, water sensitivity or high cost. Moreover, these red phosphors are formulated using rare earth elements (REE). The world's demand for REE, especially within the clean energy, military, and consumer electronics sectors, is projected to reach 200 000 tons per year by 2025, while the current annual supply is estimated at only 113000 tons. Any attempt to increase REE supply at a high enough rate to meet the demand will encounter many economic, political, and environmental limitations. ${ }^{3}$ Indeed, mining requires a high capital input due to the necessity to extract from new sites with their associated refining facilities. ${ }^{4}$ The REE mining process also produces large amounts of waste, which once released cause huge environmental damage. Economic issues are also related to political ones, since the lack of security in the supply chain creates significant instability in the REE market. Currently, over $95 \%$ of the global REE supply comes from China.

In this context, it is now crucial to identify or design REE-free phosphors capable of delivering cost-effective light energy conversion, especially for commercial WLED applications, which represent a very large market. Among the alternatives to REE containing phosphors, efforts have been made to develop new materials, such as organic molecules or quantum dots (QD). The present study is focused on revisiting an organic molecule, sulforhodamine B (SRB), a commonly-used dye. This 
choice is guided by the fact that this common organic dye, belonging to the xanthene family, emits a red-orange light $\left(\lambda_{\mathrm{em}}\right.$, $\max =590 \mathrm{~nm})$ upon green excitation $\left(\lambda_{\mathrm{abs}, \max }=565 \mathrm{~nm}\right)$ in aqueous diluted solution. Moreover, the SRB molecules are able to absorb photons in a broad spectral range from 450 to $600 \mathrm{~nm}$, which makes them compatible with blue commercial chips. Based on the optical properties of SRB, the use of SRB dye in a white LED is expected to emphasize the reddish contribution of the global emission spectra.

Until recently, most of the works published in the literature have been related to the use of SRB in cell cytotoxicity assays. $^{5-7}$ As far as LED applications are concerned, SRB molecules have never been investigated. Indeed, SRB exhibits interesting luminescence characteristics but only in diluted solutions, ${ }^{8,9}$ as most organic dyes, and is optically inactive in its solid state. Furthermore, it has already been reported that the straightforward dispersion of dye molecules into a polymer can cause them to aggregate, thus forming heterogeneous composite films presenting a tiny absolute photoluminescence quantum yield ( $\mathrm{PLQY}$ abs) ${ }^{10}$ underlining the need to maintain the dye molecules apart from each other to avoid their optical quenching. An elegant solution consists in immobilizing the SRB molecules in a host lattice like a layered double hydroxide (LDH) as previously reported. ${ }^{11}$ Pioneering research has underlined the benefit of confining photoactive species in a restricted space such as a two-dimensional host in terms of 1) their strong adsorption through host-guest interaction, to avoid possible migration and aggregation within a polymer, 2) their dispersion in the presence of co-adsorbates to avoid, as far as possible, guest-guest interactions. ${ }^{12}$

Traditionally, LDH materials have found applications as precursors in catalysis and as scavengers in environmental applications, but have also generated renewed interest in the energy field ${ }^{13,14}$ as well as in biology. ${ }^{15-17}$ Their general chemical formula is as follows $\left[\mathrm{M}(\mathrm{II})_{1-\mathrm{x}} \mathrm{M}(\mathrm{III})_{\mathrm{x}}(\mathrm{OH})_{2}\right]^{\mathrm{x}+}\left[\mathrm{A}^{\mathrm{n}-}\right]_{\mathrm{x} / \mathrm{n}} \cdot \mathrm{mH}_{2} \mathrm{O}$, where $\mathrm{M}(\mathrm{II})$ is a bivalent cation such as $\mathrm{Zn}^{2+}, \mathrm{Mg}^{2+}, \mathrm{Fe}^{2+}$, etc.; $\mathrm{M}(\mathrm{III})$ is a trivalent cation such as $\mathrm{Al}^{3+}, \mathrm{Fe}^{3+}$, etc.; and $\mathrm{A}^{\mathrm{n}-}$ is an anion. The anisotropic two-dimensional structure is built from an edge-sharing octahedral. Compared to brucite $\mathrm{Mg}(\mathrm{OH})_{2}$, the partial substitution of divalent cations by trivalent cations implies a net positive charge for LDH sheets. To ensure electroneutrality, anions of interest, here the dye molecules, are located in the interlayer space of the LDH with the presence of water molecules, concomitantly with surfactant molecules (see below).

The use of a LDH structure for LED applications has scarcely been reported so far, and mostly concerns rare-earthcontaining LDH such as Eu ${ }^{3+18}$ or $\mathrm{Tb}^{3+}$ ions $^{19}$, or QDs such as CdTe ${ }^{20}$ or core-shell InP/ZnS. ${ }^{21}$ The LDH structure could also be used as a host matrix in order to synthesise ultra-thin $2 \mathrm{D}$ carbon nitride (CN) in the interlamellar space; this material exhibits very high luminescence properties, compatible with WLED applications. ${ }^{22}$

As mentioned above, only Yan et al. have already performed the intercalation of SRB in a LDH host matrix. ${ }^{11}$ However, several studies deal with the combination of SRB and different substrates. SRB molecules have been covalently bound to core PAMAM dendrimers which then offer blue emissive species, ${ }^{23}$ whereas the SRB molecules are originally red-emitting dyes. SRB photoactive species have also been attached to silica spheres ${ }^{24}$ and adsorbed onto different pore-size silicas ${ }^{25}$ that were found to affect the absorption and emission properties of the dye with a bathochromic effect. When SRB molecules are supported on graphene oxide or boron nitride nanosheets, ${ }^{26}$ an electron-transfer process occurs, producing a non-luminescent complex with the former substrate and rapid quenching for the latter. From this rapid survey, SRB molecules seem to offer preferentially highly and stable luminescence properties when entrapped in a 2D matrix such as $\mathrm{LDH}$, rather than when combined on 1D or 3D substrates, where their luminescence is quenched or unsuitably shifted.

It is well known that the aggregation of dye molecules may cause the quenching of luminescence, arising from the dense molecular packing that forms possible guest-guest interactions which turn off the luminescence. Thus, the quantity of dye molecules dispersed onto or into a host structure should be as low as required by the optical properties. Experimentally, the dye molecules are dispersed with surfactant molecules such as dodecylsulfate (DS) or dodecylbenzenesulfonate (DBS) when using a LDH host structure, since the surfactant acts as a spacer enabling the dye molecules to be well accommodated and ensconced within the host galleries. ${ }^{10,12,27,28}$ Intentionally, a small amount of SRB was used in $\mathrm{Zn}_{2} \mathrm{Al}(\mathrm{OH})_{6}(\mathrm{DS})_{1-\mathrm{x}}(\mathrm{SRB})_{\mathrm{x}} \cdot \mathrm{nH}_{2} \mathrm{O}$, and the domain investigated here, $0.0003 \leq \mathrm{x} \leq 0.02$ has a much smaller SRB relative value range than previously reported in $\mathrm{Mg}_{3} \mathrm{Al}(\mathrm{OH})_{8}(\mathrm{DBS})_{1-\mathrm{x}}(\mathrm{SRB})_{\mathrm{x}} \cdot \mathrm{nH}_{2} \mathrm{O}$ (with $\left.0.06 \leq \mathrm{x} \leq 1 \mathrm{in}\right){ }^{11}$

Another aspect investigated here for LED applications concerns the protection of the photoactive molecules against possible thermal degradation and photonic stress occurring when used in the LED set-up.

In this study, the intercalation of SRB molecules into a $\mathrm{Zn}_{2} \mathrm{Al}$ LDH host structure was performed using the usual coprecipitation method. To dispose SRB molecules as far as possible from each other dodecylsulfate surfactant molecules 
(DS) were co-intercalated with SRB. Indeed, the surfactant increases gallery height, which facilitates the incorporation of the SRB molecules, and at the same time its long alkyl chains supply a non-polar environment, preventing the SRB molecules from aggregating. Optical characterizations of the LDH-DS-SRB were investigated using IR and UV spectroscopy, and $\mathrm{PL} \mathrm{QY} \mathrm{Ybs}_{\mathrm{as}}$, emission spectra and CIE chromaticity coordinates were also recorded. Luminescent composite films prepared from LDH-DS-SRB dispersed in silicone were combined with commercial blue chip and YAG:Ce phosphor to produce a warm white light. Finally, the stability under thermal and photonic stresses of such composite films was compared to that of a silicone/LDH-free SRB film to demonstrate the benefits of such a hybrid co-intercalated LDH-DS-SRB assembly.

\section{Experimental}

\section{Materials}

Sulforhodamine $B$ sodium salt $\mathrm{C}_{27} \mathrm{H}_{29} \mathrm{~N}_{2} \mathrm{NaO}_{7} \mathrm{~S}_{2}$ and sodium dodecylsulfate $\mathrm{CH}_{3}\left(\mathrm{CH}_{2}\right)_{11} \mathrm{OSO}_{3} \mathrm{Na}$ were purchased from Sigma-Aldrich, and YAG:Ce ${ }^{3+}$ from Phosphortech. $\mathrm{Zn}\left(\mathrm{NO}_{3}\right)_{2} .6 \mathrm{H}_{2} \mathrm{O}$ (purity $\left.99.9+\%\right), \mathrm{Al}\left(\mathrm{NO}_{3}\right)_{3} .9 \mathrm{H}_{2} \mathrm{O}$ (purity $\left.99.9+\%\right)$ and $\mathrm{NaOH}$ (97\%) were obtained from Sigma Aldrich. The two-component silicone elastomer, Bluesil RTV 141 part A and part B, was supplied by Elkem.

\section{Synthesis procedure of LDH hybrid materials using the coprecipitation method}

The series of sulforhodamine B (SRB) -dodecyl sulfate (DS) LDH phases, Zn $n_{2} A$ I-DS-SRB, called LDH-DS-SRB, was prepared by coprecipitation with various $\mathrm{DS} / \mathrm{SRB}$ ratios. The synthesis of $\left[\mathrm{Zn}_{2} \mathrm{Al}_{1}(\mathrm{OH})_{6}\right]^{+}\left[\mathrm{DSS}^{-}\right]_{1-\mathrm{x}}\left[\mathrm{SRB}^{-}\right]_{x} \cdot \mathrm{mH}_{2} \mathrm{O}$ was performed using 50 $\mathrm{mL}$ of sulforhodamine $B$ and dodecylsulfate aqueous solution (with de-ionized water). $50 \mathrm{~mL}$ of an aqueous solution of $\mathrm{Zn}^{2+}$ $(3.2 \mathrm{mmol})$ and $\mathrm{Al}^{+3}(1.6 \mathrm{mmol})$ was added dropwise over a period of 3 hours under magnetic stirring. For instance, to synthesize the $\left[\mathrm{Zn}_{2} \mathrm{Al}_{1}(\mathrm{OH})_{6}\right]^{+}\left[\mathrm{DS}^{-}\right]_{0.999}\left[\mathrm{SRB}^{-}\right]_{0.001} \cdot \mathrm{mH}_{2} \mathrm{O}$ sample, $1.6 \times 10^{-3} \mathrm{mmol}$ and $1.5984 \mathrm{mmol}$ were used for SRB salt and sodium dodecylsulfate, respectively.

The $\mathrm{pH}$ was maintained at 8.5 by adding $0.25 \mathrm{M} \mathrm{NaOH}$ during the synthesis process. Coprecipitation was performed under nitrogen at $20^{\circ} \mathrm{C}$. The mixture was centrifuged at 5,000 rpm for 5 minutes; the sedimented solid on the bottom of the flask was washed several times with de-ionized water until a clear and transparent supernatant was obtained. The paste was recovered and dried overnight at room temperature to obtain a powder.

\section{Manufacture of Silicone/HDL-DS-SRB composite films}

The LDH-DS-SRB powder was used to produce the silicone/hybrid composite material with different hybrid loadings. The two-component silicone elastomer (silicone Bluesil-RTV $141 \mathrm{~A} \& \mathrm{~B}$ ) was composed of a viscous liquid, called part A, cured by a polyaddition reaction with a catalyser, part $\mathrm{B}$.

The silicone film was prepared as follows: a mixture of part A and part B (10 phr of part A) was homogenised using a mechanical mixer (Planetary Centrifugal vacuum Mixer "Thinky Mixer") for $10 \mathrm{~min}$ at $1200 \mathrm{rpm}$.

The silicone/hybrid composite material (20 wt. \% LDH-DS-SRB and $80 \mathrm{wt}$. \% silicone) was made by mixing the $\mathrm{LDH}$ powder $(0.6 \mathrm{~g})$ with part A of the silicone elastomer $(2.18 \mathrm{~g})$ using the mechanical mixer ("Thinky Mixer") for $10 \mathrm{~min}$ at $1200 \mathrm{rpm}$. Then the mixture obtained was passed through a rolling mill (EXAKT brand) composed of three rollers (spacing of $30 \mu \mathrm{m}$ between the first two rollers and $50 \mu \mathrm{m}$ between the last two rollers) to achieve a better dispersion of the LDH platelets by shearing and to obtain a homogeneous hybrid component. Part B was added at $10 \mathrm{phr}(0.22 \mathrm{~g})$ and homogenised using the mechanical mixer for $10 \mathrm{~min}$ at $1200 \mathrm{rpm}$. The silicone film and the silicone/hybrid composite film were prepared by casting onto a Teflon surface using an Elcometer 4340 automatic film applicator. The knife blade height was set to $200 \mu \mathrm{m}$ and the casting speed was $30 \mathrm{~mm} / \mathrm{s}$. This twocomponent silicone film was cured at $80^{\circ} \mathrm{C}$ for 2 hours. After curing, the film thickness $(90 \pm 5 \mu \mathrm{m})$ was measured using an Elcometer 456 coating thickness gauge.

\section{Characterisation}

\section{X-ray diffraction}

LDH powders were characterised by X-Ray Diffraction; the XRD patterns were recorded with a Philips X-Pert Pro diffractometer operating with Cu-Ka radiation $\left(\lambda=1.5418 \AA\right.$ ) . The data were collected in a $2 \theta$ range between $5^{\circ}$ and $70^{\circ}$ with a step size of $1 \%$ min.

\section{Thermogravimetric analysis}

Thermogravimetric (TG) analyses were performed on a Setaram TGA 92 instrument with a linear heating rate of 5 ${ }^{\circ} \mathrm{C} \cdot \mathrm{min}^{-1}$ under air. 


\section{UV-Visible absorption}

The UV-visible absorption spectra of the samples were recorded in the wavelength range of 200 to $800 \mathrm{~nm}$ with a UVvis spectrophotometer (SP-3000 Plus) equipped with an integrating sphere and UV-Probe software.

\section{Luminescence}

Quantum yield efficiencies and emission spectra were measured using a C9920-02G PL-QY integrating sphere measurement system from Hamamatsu Photonics. The setup consisted of a $150 \mathrm{~W}$ monochromatized Xe lamp, an integrating sphere (Spectralon coating, $\varnothing=3.3 \mathrm{in}$.) and a high-sensitivity CCD camera.

\section{Emission stability of films under LED irradiation}

Reliability studies were carried out using a home-made setup consisting of a power-controlled blue LED emitting at 450 $\mathrm{nm}$ as the excitation source, and a HR4000 high resolution spectrometer (Ocean Optics) as the PL analyser. The samples were positioned on a heating element whose temperature was adjusted to $80{ }^{\circ} \mathrm{C}$. The emission spectra of the composite films were collected every 20 min over a period of $24 \mathrm{~h}$. Their area was integrated to obtain the total emission intensity. LED power was measured using a Scientech Model Mentor MA 10 with a MC2501 calorimetric head unit $(25.4 \mathrm{~mm}$ aperture). Measurement was performed by centring the head unit over the LED source and measuring the LED power of light emitted through the aperture. The blue LED power was $183 \mathrm{~mW}$. The LED power density expressed in W/m² was calculated by LED power (in watt) per unit surface of the sample $\left(0.25 \mathrm{~cm}^{2}\right)$. According to our experiment, the LED power density was 2480 W.m $\mathrm{m}^{-2}$ for blue LED.

\section{Results and discussion}

\section{Concentration screening of SRB in LDH matrix}

XRD pattern
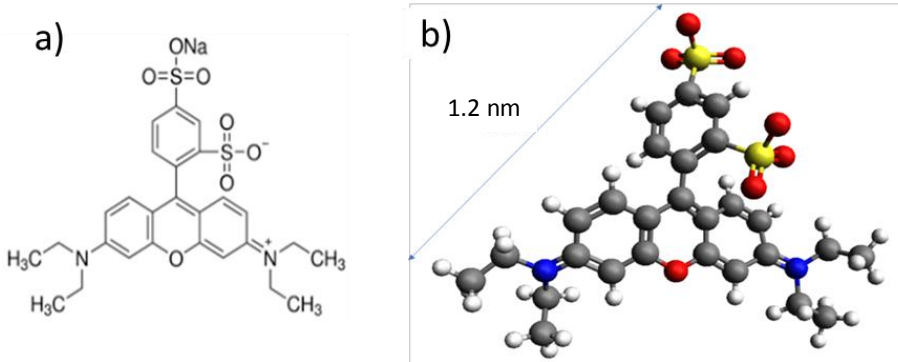

Figure 1. Schematic representation of SRB dye in 2D (a) and 3D (b).

SRB (Fig. 1 a) and b)) is an organic dye composed of a planar xanthene backbone with a benzenedisulfonate group.

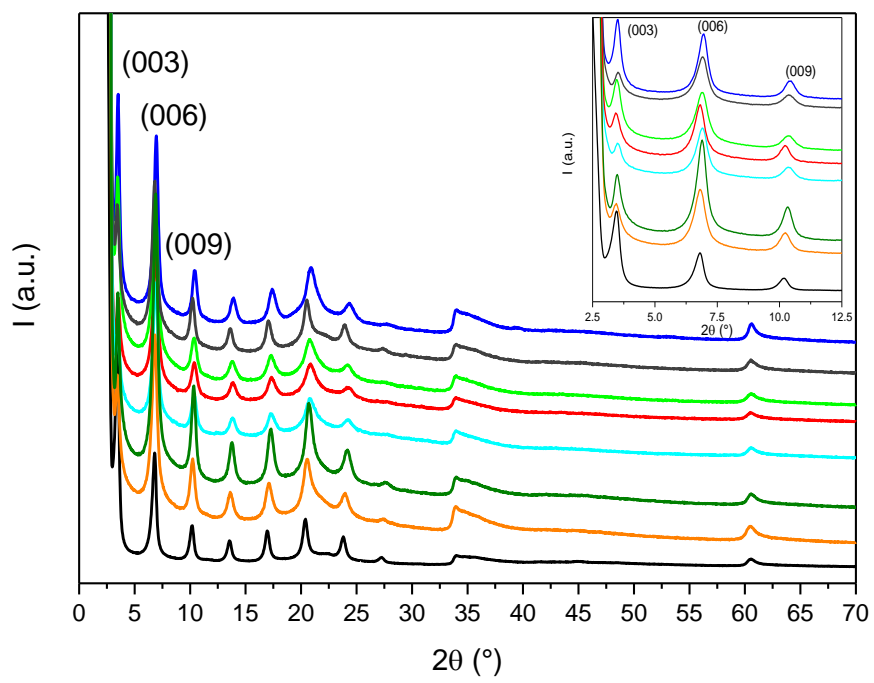

Figure 2. XRD patterns between 0 and $70^{\circ}$ and between 2.5 and $12.5^{\circ}$ (insert) of samples synthetized by varying molar ratio $x$, from bottom to top $x$ $=0.02 ; 0.01 ; 0.005 ; 0.002 .0 .001 ; 0.0008 ; 0.0005 ; 0.0003$ and 0 .

As mentioned in the introduction, this kind of photoactive molecule is known for its efficient incorporation into LDH matrices. ${ }^{3,11}$ The dispersion provided by hosting photoactive molecules in LDH has already been demonstrated ${ }^{10}$ to improve 
the luminescent properties of dyes such as fluorescein. In the same vein, the hosting of SRB molecules in $\mathrm{Zn}_{2} \mathrm{Al}$ is expected to lead to a luminescent hybrid powder. Additionally, the DS spacer is here co-intercalated to further homogeneously disperse SRB molecules within the inter-lamellar space. The DS surfactant is known to hold apart the stacked layers that could subsequently be of interest when such a hybrid assembly is used as filler and dispersed into a polymer.

Firstly, the optimal DS/SRB ratio leading to the best luminescence properties was established through the absolute photoluminescence quantum yield values $(\mathrm{PL} \mathrm{QY}$ abs $)$. To this aim several syntheses were performed, varying the molar ratio $x=n_{S R B} /\left(n_{D S}+n_{S R B}\right)$ with $0.0005<x<0.02$. The XRD patterns between 2 and $70^{\circ}$ in $2 \theta$ of each LDH-DS-SRB sample made, as well as the template LDH-DS powder, are presented in Fig. 2. Series of harmonic peaks (0OI) are well observed (Fig 2. insert) and result in a basal spacing of $2.5 \mathrm{~nm}$, in agreement with the literature for LDH/DS. ${ }^{29}$ However, the SRB content is too small to be observed, the basal spacing being imposed by the spacer alone.

\section{Absolute Photoluminescence Quantum Yields}

Variations in the absolute photoluminescence quantum yields ( $\left.P L \mathrm{QY}_{\mathrm{abs}}\right)$ as a function of the excitation wavelengths are shown for each sample in Fig. 3a.

Three wavelengths are of interest: $365 \mathrm{~nm}$ and $480 \mathrm{~nm}$ wavelengths correspond to UV and blue commercial

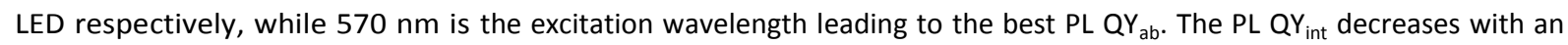
increase in SRB content, whereas Absorbance increases with the SRB amount for both excitation wavelengths, 480 and 385

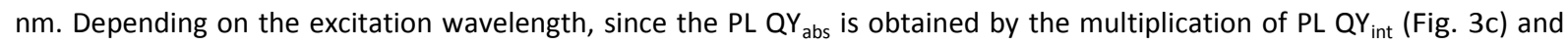
Absorbance (Fig. 3d), a maximum of PL QY $Y_{\text {abs }}$ is reached for $x_{S R B}=0.0005$ with a value close to $60 \%$ for $\lambda_{\text {exc }}=570 \mathrm{~nm}$, whereas for $\lambda_{\mathrm{exc}}=365 \mathrm{~nm}$ and $480 \mathrm{~nm}$ the maximum of $\mathrm{PL} \mathrm{QY_{ \textrm {abs } }}$ corresponds to $\mathrm{x}_{\mathrm{SRB}}=0.002$.

Fig. 4 illustrates the variation in PL QY ${ }_{\text {abs }}$ as a function of the SRB amount in the LDH host structure for $\lambda_{\text {exc }}=480 \mathrm{~nm}$.

For $\lambda_{\text {exc }}=480 \mathrm{~nm}, \mathrm{PL} \mathrm{QY}$ abs luminescence decreases when the SRB amount becomes higher than $\mathrm{x}=0.002$. This observation could be explained by a concentration quenching phenomenon, which is predominant when the concentration of SRB increases above a certain threshold. The same behaviour was evinced in the case of fluorescein molecules incorporated into LDH galleries. ${ }^{10}$ Indeed, when intercalated SRB molecules are sufficiently close to each other in such a restricted space, they are prone to aggregation by $\pi$-stacking (parallel stacking) due to conjugated xanthene rings or by hydrogen bonding (head-to-tail). Xanthene dyes such as SRB or fluorescein are well-known ${ }^{30}$ to interact mutually, resulting
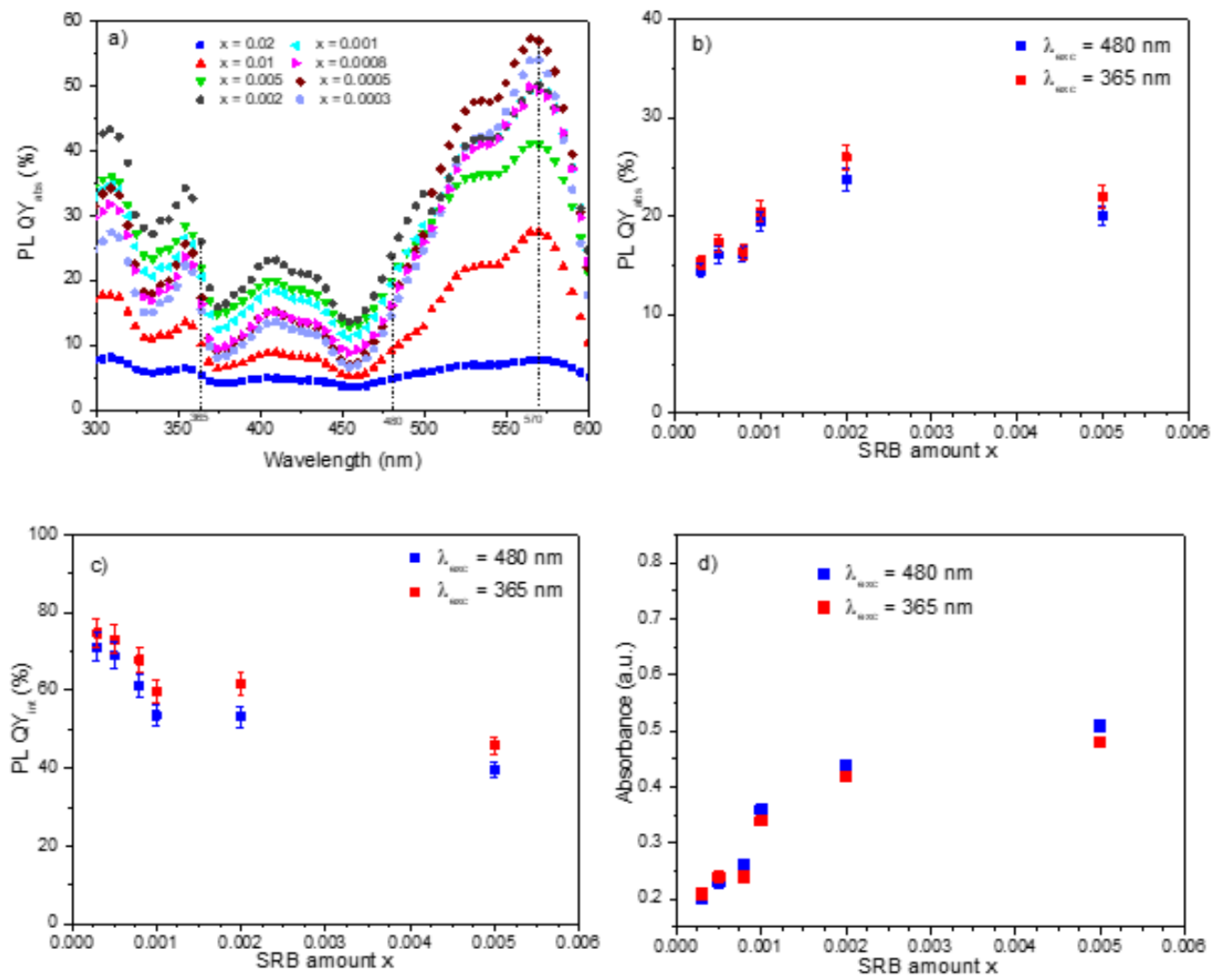

Figure 3. (a) Variation in PL QY abs as a function of the excitation wavelength for LDH-DS-SRB samples and variation in $\mathrm{PL} \mathrm{QY}$ abs (b), internal photoluminescence quantum yield $\mathrm{PL} \mathrm{QY}$ int $(\mathrm{c})$ and Absorbance (d) as a function of the SRB content $(x)$ for $\lambda_{\mathrm{xc}}=480 \mathrm{~nm}$ and $\lambda_{\mathrm{ec}}=365 \mathrm{~nm}$. 
in luminescence quenching when the concentration reaches a critical value to form dimers, trimers, etc.

Although the monomer yields luminescence properties, most aggregates led to the extinction of the optical properties due to non-radiative energy transfer between the dye molecules. ${ }^{31}$ This phenomenon increases with the dye concentration in solution, and luminescence totally disappears for SRB powder. The restricted space supplied by the LDH host reduces the SRB molecular packing and acts as a dilute medium in dispersing the SRB dyes. An optimal value of SRB of $x=0.002$ is found, corresponding to about 25 times less than the values previously reported; ${ }^{11}$ all the reported values were surprisingly high, corresponding here to the extinction of luminescence for the SRB molecules.

All the excitation wavelengths studied result in an optimal PL QYabs for a very low concentration of photoactive molecules in the LDH inter-lamellar space. In all cases, a compromise between a high internal photoluminescence quantum yield (isolated SRB molecules in the inter-lamellar-space) while maintaining an appropriate absorbance (given for a significant amount of SRB in the LDH-DS-SRB samples) must be reached (Fig. 3).

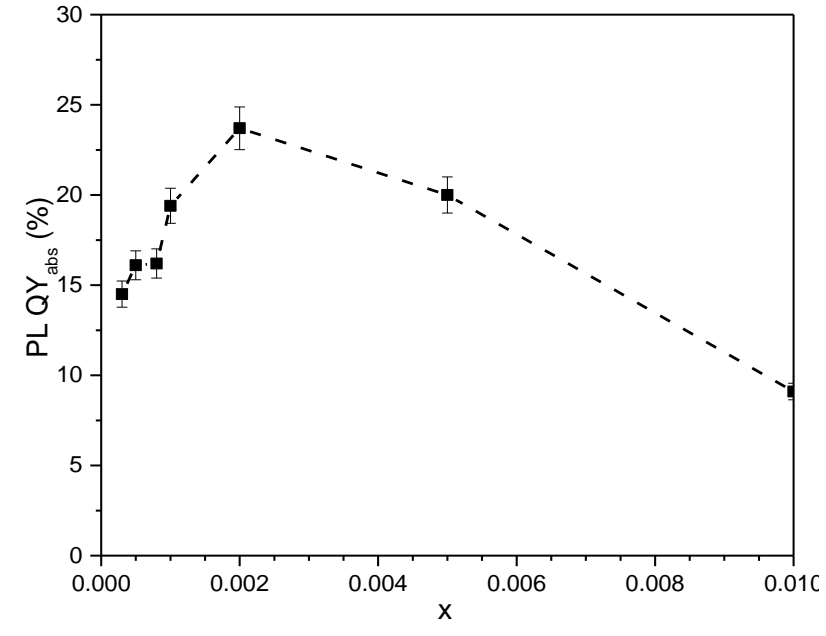

Figure 4. Variation in PL QYabs as a function of $x$ for $\lambda_{\mathrm{exc}}=480 \mathrm{~nm}$.

\section{Optical properties}

In the following section, the optical properties of the LDH-DS-SRB sample with the optimized luminescence properties under blue excitation $(480 \mathrm{~nm})$, LDH-DS-SRB $(x=0.002)$, defined as LDS in composite are presented.

\section{Absorption properties}

In order to study the absorption of the SRB molecules in the LDH-DS-SRB powder, the absorption spectrum was recorded in solid state through an LDH-DS-SRB-KBr pellet. This was prepared by the dispersion of the pristine LDH-DS-SRB $\left(0.01 \%_{w}\right)$ in $\mathrm{KBr}$ powder. The UV-visible absorption spectrum obtained for the pellet is presented in Fig.5 and compared to that of SRB in aqueous solution $\left(10^{-4} \mathrm{M}\right)$. As reported by several authors such as K. Ray et al. ${ }^{32}$, V. Paolucci et al. ${ }^{23}$ or Wittman et Penzkofer ${ }^{33}$, in dilute aqueous or alcohol solutions, the absorption spectrum of SRB is characterised by a typical profile, as shown in Fig. 5 (blue line). Indeed, the absorption band at $575 \mathrm{~nm}$ is linked to the $\mathrm{S}_{0}-\mathrm{S}_{1}$ absorption of monomers, whereas the obvious shoulder at $535 \mathrm{~nm}$ comes from the $\mathrm{S}_{0}-\mathrm{S}_{1}$ absorption of aggregates (dimer, trimer...) ${ }^{34}$. Regarding the UV-Visible absorption spectrum of LDH-DS-SRB powder, all the distinctive absorption bands of SRB molecules are observed. However, the monomer/aggregate ratio increases significantly, due to the presence of numerous aggregates in the LDH-DSSRB powder, where the dilution of SRB is worse than in the aqueous solution $\left(10^{-4} \mathrm{M}\right)$. Other absorption bands appear between 200 and $450 \mathrm{~nm}$, and can be assigned to the absorption of photons from the fundamental state to more energetic $\mathrm{S}_{\mathrm{n}}$ excited states. $^{35}$

Furthermore, an increase in absorption between 200 and $250 \mathrm{~nm}$ is attributed to the absorption of $\mathrm{Zn}^{2+}$ and $\mathrm{Al}^{3+}$ cations from the LDH matrix. ${ }^{36}$ Thus, absorption properties are not modified but less intense after SRB intercalation. 


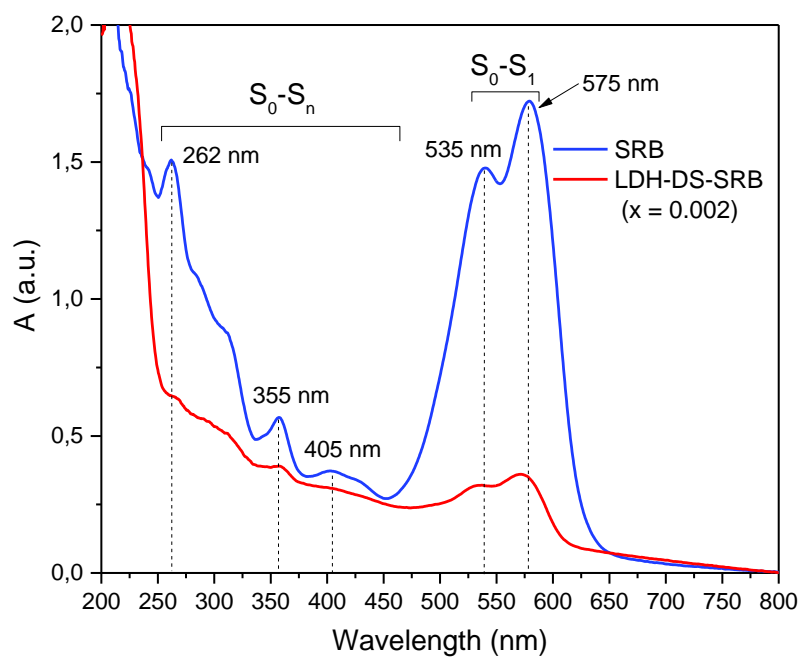

Figure 5. Absorption spectra of SRB in aqueous solution $\left(10^{-4} \mathrm{M}\right)$ (blue line) and of the LDH-DS-SRB powder $(x=0.002)($ red line).

\section{Emission spectra}

The normalized emission spectra of LDH-DS-SRB powder and SRB in aqueous solution $\left(10^{-4} \mathrm{M}\right)$ were recorded under blue excitation $(480 \mathrm{~nm})$ as shown in Fig. 6 . The emission spectrum of SRB exhibits a broad and asymmetric band between 550 and $750 \mathrm{~nm}$, with a maximum at $625 \mathrm{~nm}$ and a large shoulder at around $655 \mathrm{~nm}$. As described by Yan et al. [4], these contributions are related to different environments for SRB molecules. The main peak located at the highest energy point corresponds to the SRB monomers, while the shoulder is attributed to the aggregated forms (dimers, trimers, ...). Compared to the LDH-DS-SRB matrix, the emission spectrum (Fig. 6, line red) has a similar profile. However, the fluorescence maximum is red-shifted (by $30 \mathrm{~nm}$ ) and the ratio between the two main emission bands is modified. Indeed, the contribution to the longer wavelengths increases. We can conclude from these observations that the aggregated forms of SRB are in slightly greater quantity for SRB in the LDH matrix compared to SRB in dilute aqueous solution $\left(10^{-4} \mathrm{M}\right)$.

These aggregated forms of SRB are responsible for non-radiative de-excitation processes that are accompanied by a decrease in light emission properties. Similar behaviour has been identified for the intercalation of fluorescein into a LDH host with the formation of J-type and H-type aggregates. ${ }^{10}$

As shown in Fig. 6b), LDH-DS-SRB powder $(x=0.002)$ and SRB in solution both exhibit CIE colour coordinates associated with a red-orange emission. In accordance with its emission spectrum, the sample leads to a more reddish colouring than the SRB solution.

The SRB content in the LDH host matrix is clearly shifting the coordinates (x;y) in the CIE 1931 plot of each sample (Fig. S1). An increase in SRB input tends to accentuate the red contribution of the emitted light under blue excitation. This behaviour is also observed when the SRB molecules are encapsulated in silica spheres ${ }^{24}$, due to aggregation effects, whereas when the SRB molecules are attached to PAMAM dendrimers, ${ }^{23}$ the emission is then blue-shifted. In this latter case, the luminescence mechanism is modified because of electron transfers between the dye molecules and the core dendrimers.

\section{Composite silicone/LDH-DS-SRB film $(x=0.002)$}

To further assess the possible interest of the selected LDH-DS-SRB $(x=0.002)$ powder for a LED application, a composite film was prepared by dispersing the powder in a silicone elastomer (PDMS-type) matrix (according to the protocol described in the experimental part). A composite film, named Si-LDS, with a thickness of $90 \pm 5 \mu \mathrm{m}$ was obtained by mixing 20 wt. \% LDH-DS-SRB $(x=0.002)$ and $80 \mathrm{wt}$. \% silicone. The photographs of this composite film are reported on Fig. S2 under daylight and UV radiation (365 nm) showing the optical properties. The SEM image (Fig. S3) of the film confirms the homogenous dispersion of the particles (LDH-DS-SRB) in the elastomer. The emission spectrum of the Si-LDS composite film (Fig. 6a) was recorded under excitation at $480 \mathrm{~nm}$. Comparison of this spectrum with that of the LDH-DS-SRB powder $(x=$ 0.002 ) shows a shift towards shorter wavelengths. This phenomenon can be explained by a disaggregation effect of the LDH platelets, and consequently by an increase in the distance between SRB molecules induced by the rolling step during film preparation. Moreover, the contribution at the longest wavelengths previously attributed to the aggregated forms of SRB is less intense in the film than in the powder. Both observations agree with the fact that the fabrication process of the composite film allows the SRB molecules to disaggregate, thus providing an additional dilution effect by reducing the nonradiative energy transfers. 
As a result, the chromaticity diagram exhibits a slightly more orange-coloured dot for Si-LDS film than for LDH-DS-SRB powder (Fig. 6b).
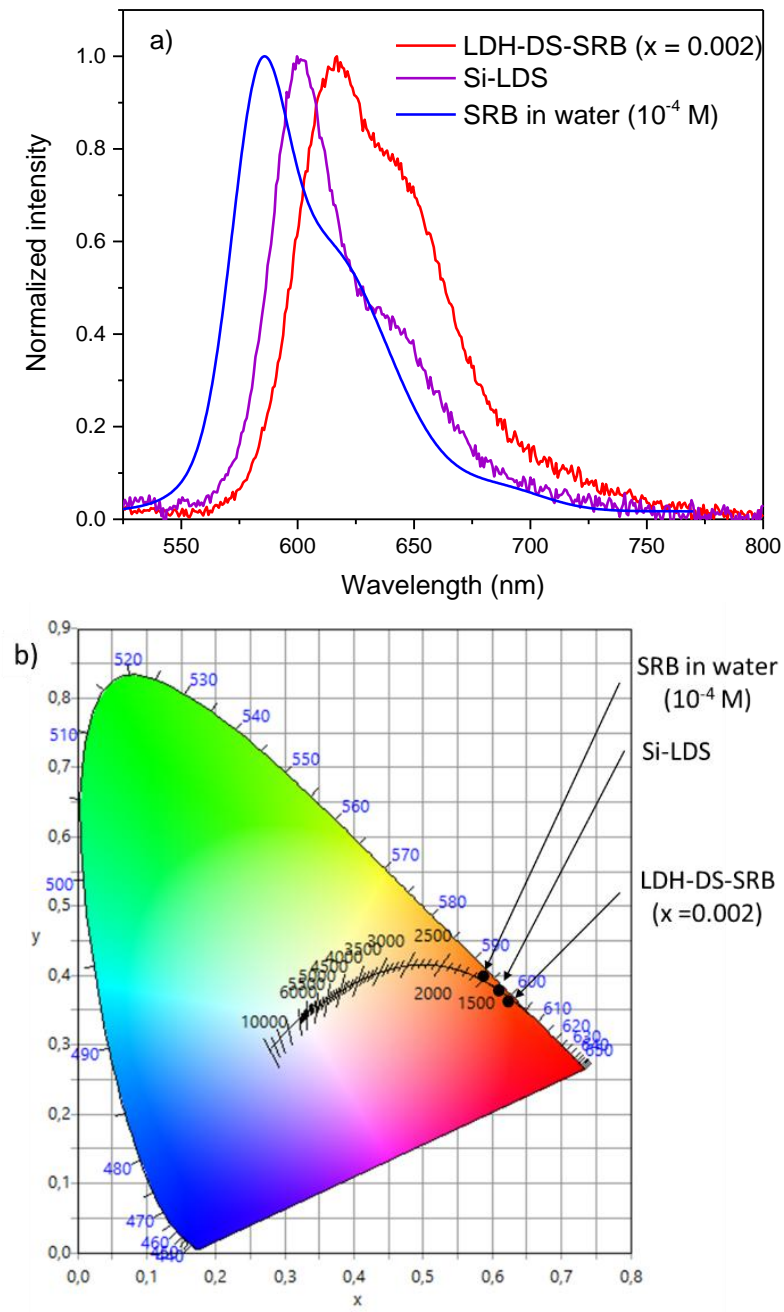

Figure 6. a) Emission spectra of the LDH-DS-SRB powder $(x=0.002)$ (red line), SRB in water solution (10 $\left.{ }^{-4} \mathrm{M}\right)$ (blue line) and the silicone Si-LDS film (purple line); b) CIE chromaticity coordinates of LDH-DS-SRB powder $(x=0.002)$, SRB in water $\left(10^{-4} \mathrm{M}\right)$ and Si-LDS composite film

\section{PL QYabs of Si-LDS composite film}

$P L Q Y_{a b s}$ of the Si-LDS composite film were recorded for excitation wavelengths from 300 to $600 \mathrm{~nm}$ (Fig. 7). The film exhibits the same values as for the LDH-DS-SRB $(x=0.002)$ powder, meaning that the dispersion in silicone did not modify photoluminescence behaviour. For the composite film, the decrease in absorbance by dispersing the LDH-DS-SRB ( $x=$ 0.002) powder is counter-balanced by a decrease in the non-radiative energy transfers, thus explaining why similar PL QY abs $_{\text {s. }}$ values are obtained (Fig. S4). 


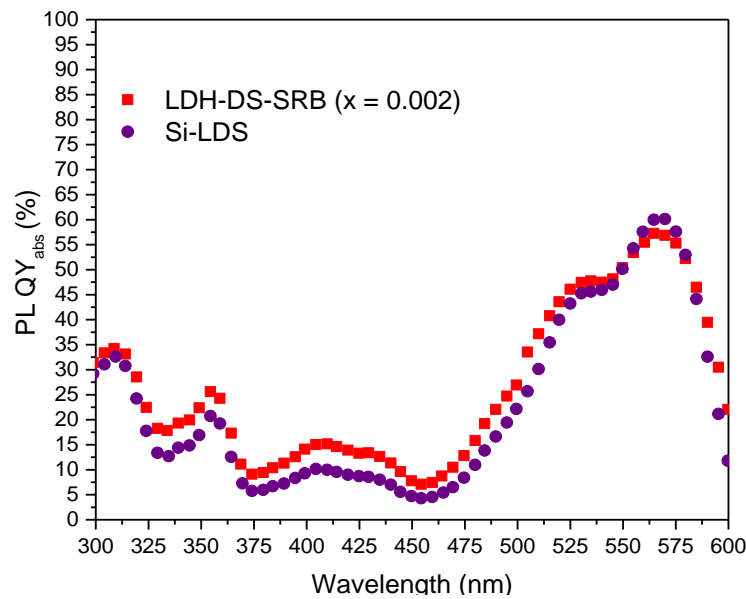

Figure 7. Variation in PL QY $\mathrm{abs}_{\text {for }}$ fhe Si-LDS composite film and the LDH-DS-SRB $(x=0.002)$ powder.

\section{White light emission produced by the association of a blue LED chip and two silicone films}

As shown previously, the emission spectrum of LDH-DS-SRB covers a wide wavelength range from yellow to red. It is of interest to extend the yellow emission of the commercial phosphor YAG:Ce (Fig. 8) to the red wavelength range. Thus the combination of a blue LED chip with these two phosphors, YAG:Ce and LDH-DS-SRB, is expected to yield a warmer white emission associated with a better CRI.

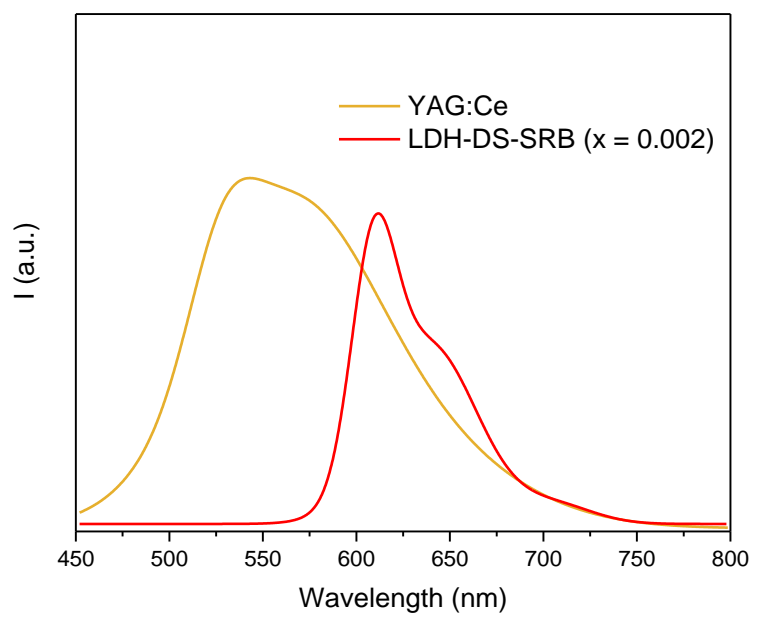

Figure 8. Emission spectra of LDH-DS-SRB $(x=0.002)$ powder and a commercial phosphor YAG:Ce $\left(\lambda_{\mathrm{exc}}=480 \mathrm{~nm}\right)$.

In order to assess this combination, a YAG-Ce composite film with a $200 \mu \mathrm{m}$ thickness (labelled Si-YAG) composed of 43 wt. \% commercial powder and $57 \mathrm{wt}$ \% bi-component silicone matrix was produced. This loading rate was optimised in previous work. ${ }^{37}$ Si-LDS and Si-YAG films were placed on a $450 \mathrm{~nm}$ commercial LED in remote-phosphor configuration as shown in Fig. 9.

The photometric parameters of three different configurations were recorded:

- $\quad$ Blue LED /Si-YAG (Fig. 9 b).

- Blue LED/Si-YAG/Si-LDS (Fig. 9 c).

- Blue LED/Si-LDS/Si-YAG (Fig. 9 d).

The emission spectra of these three configurations, as well as a picture of the device, are presented in Fig. 9. For the first LED/Si-YAG configuration, part of the contribution of the blue LED is not absorbed by the YAG: Ce composite Si-YAG film, and is transmitted (around $425 \mathrm{~nm}$ ); the composite film also emits a large contribution. The colour of the resulting light is yellow-green, which is confirmed by the CIE 1931 coordinates (Fig. S5). When the Si-LDS composite film is added above the Si-YAG film, the presence of the SRB increases the red-orange range contribution in the global emission spectrum (Fig. 9 C), and the photometric parameters of the resulting light are modified, as reported in Table 1. However, when the Si- 
LDS composite film is located between the blue LED chip and the Si-YAG film, the global spectrum emission of the system is slightly different. Indeed, as we have shown in Fig. 5, green radiations are strongly absorbed by the SRB molecules, whereas blue radiations are moderately absorbed. Thus, in the LED/Si-YAG/Si-LDS configuration, the Si-LDS composite film absorbs part of the green emission from the Si-YAG composite film, which reduces the contribution of the latter, while in the LED/SiLDS/Si-YAG configuration the re-absorption phenomenon is minimised and the contribution of the Si-YAG composite film is relatively higher.
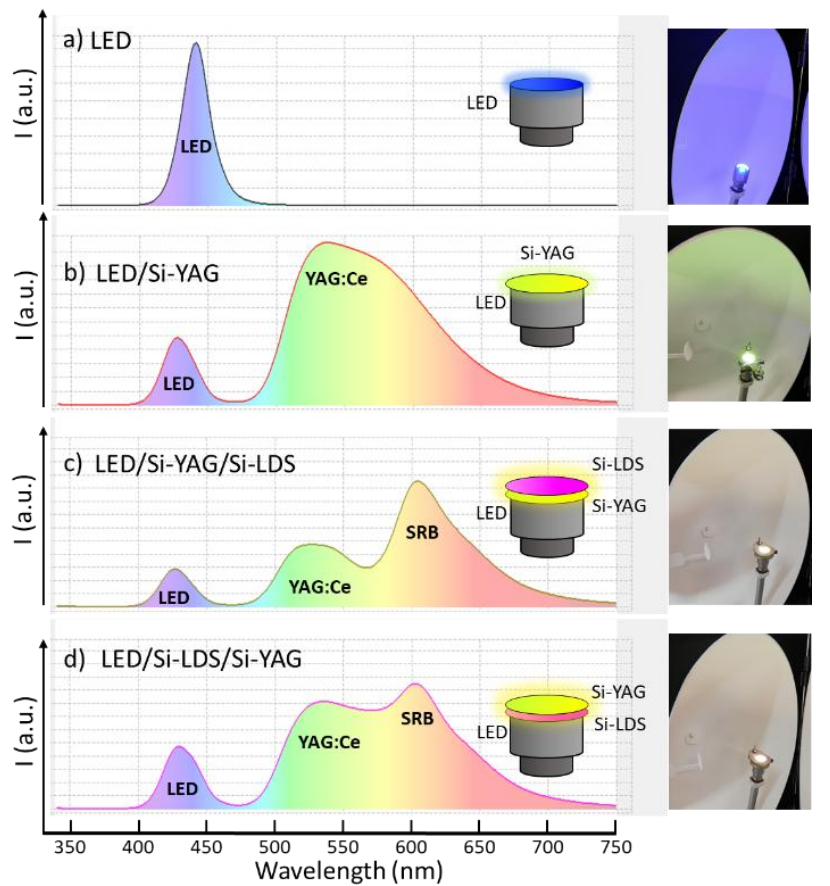

Figure 9. Emission spectra of a) commercial blue LED, and white LED prototypes designed by combinations of a commercial blue LED (450 $\mathrm{n} m$ ) with b) Si-YAG and c) and d) Si-LDS composite films.

The positions of CIE 1931 coordinates of these three configurations are presented in Fig. S5. The benefit of the SRB in the blue LED + YAG:Ce ${ }^{3+}$ system is clearly evidenced, since the position is shifted towards the well-known «blackbody» ${ }^{38}$ curve, which leads to warmer white light emission as reported in Table 1.

The colour temperature and the colour rendering index (CRI) were also recorded for each of the configurations shown in Fig. 9. It is found that both parameters are tuneable with respect to the film stacking order. Indeed, the configuration relative to Figure $9 \mathrm{c}$ ) yields a warm white with an excellent CRI. Furthermore, it underlines that a CRI close to 90 is possible to achieve, due to the strong contribution in the red spectral range provided by the Si-LDS film.

Table 1. (x:y) coordinates, correlated colour temperature (CCT) and colour rendering index (CRI) of the three presented configurations.

\begin{tabular}{|c|c|c|c|}
\hline Configuration & $\mathrm{x}: \mathrm{y}$ & $\mathrm{CCT}(\mathrm{K})$ & $\mathrm{CRI}$ \\
\hline $\begin{array}{c}\text { Blue-LED/Si-YAG } \\
\text { Blue-LED/Si-YAG/Si- } \\
\text { LDS }\end{array}$ & $0.39: 0.50$ & 4440 & 58.2 \\
\hline $\begin{array}{c}\text { Blue-LED/Si-LDS/Si- } \\
\text { YAG }\end{array}$ & $0.42: 0.45$ & weutral white & \\
\hline
\end{tabular}




\section{Si-LDS composite film stability}

In order to demonstrate the benefit of the hybrid LDH-DS-SRB powder in white LED devices, the stability of the Si-LDS composite film was studied under thermal and photonic stresses. Fig 10 shows the variations in PL QY int $_{\text {( }}$ ) as a function of the excitation wavelength for Si-LDS films before and after thermal treatment for $24 \mathrm{~h}$ at $80^{\circ} \mathrm{C}$ and $120^{\circ} \mathrm{C}$.

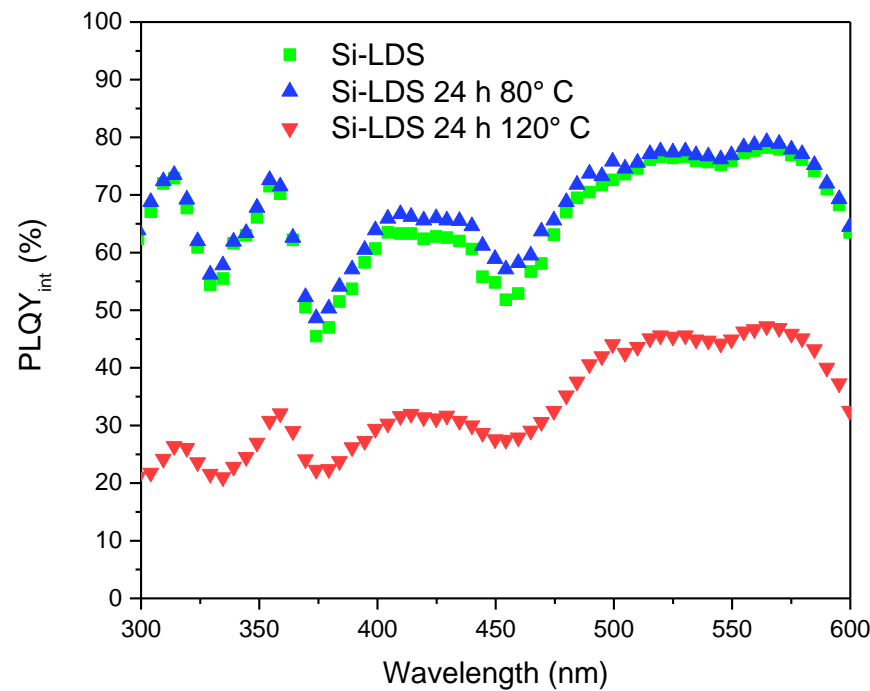

Figure 10. Variations in $\mathrm{PL} \mathrm{QY}$ int as a function of the excitation wavelength for Si-LDS film and Si-LDS films heat-treated at $80{ }^{\circ} \mathrm{C}$ and $120^{\circ} \mathrm{C}$ for 24 hours.

Under $480 \mathrm{~nm}$ excitation (wavelength of a blue commercial chip), the Si-LDS film heat-treated $24 \mathrm{~h}$ at $120^{\circ} \mathrm{C}$ lost approximately $43 \%$ of its initial $\mathrm{PL} \mathrm{QY}$ int, while it remained unchanged after exposure at $80{ }^{\circ} \mathrm{C}$. As shown by the interpretation of the UV-Visible and infrared spectra of a silicone film before and after thermal treatment at $120^{\circ} \mathrm{C}$, the polymer itself is not damaged under such thermal stress (Fig. S6). Hence, we can conclude that the reduction in optical properties should be correlated to a modification of the environment of the SRB molecules. Indeed, as the temperature increases, the SRB molecules in the Si-LDS film tend to aggregate, which promotes non- radiative de-excitation processes and reduces optical performance. This phenomenon is highlighted by the emission spectra in Fig. 11.

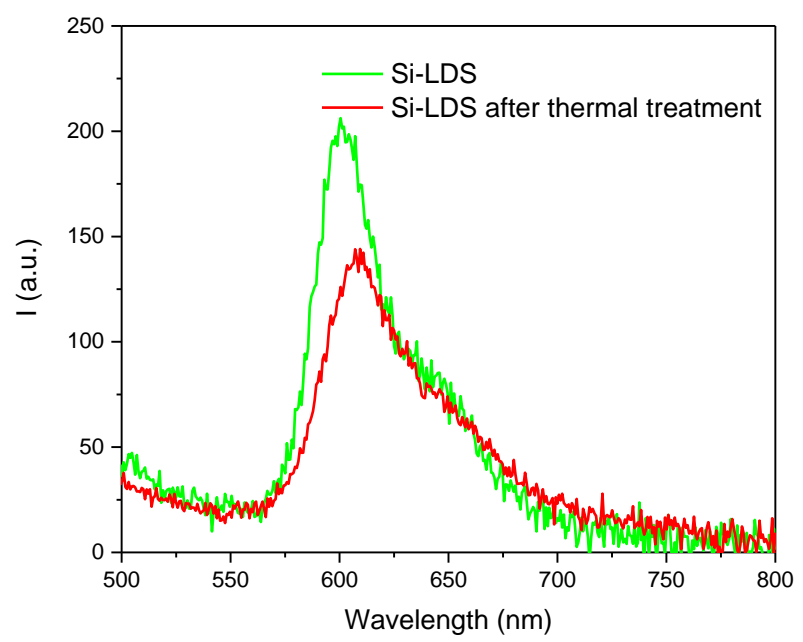

Figure 11. Emission spectra of Si-LDS films before and after thermal treatment for $24 \mathrm{~h}$ at $120^{\circ} \mathrm{C}\left(\lambda_{\text {exc }}=480 \mathrm{~nm}\right)$.

In order to assess the stability of the Si-LDS composite films in operating conditions close to those encountered in LED devices, these latter, as well as a composite film made with pristine SRB dye dispersed in a silicone matrix (Si-SRB), were irradiated by a powerful blue LED $\left(450 \mathrm{~nm}\right.$, power: $\left.2480 \mathrm{~W} \cdot \mathrm{m}^{-2}\right)$ for 1400 minutes at $80{ }^{\circ} \mathrm{C}$ in a black chamber. The amount of SRB was identical in both samples. Emission spectra were recorded every 20 minutes, and the variations in emission spectra were plotted as a function of the irradiation time in Fig. 12 for both composites. The photoluminescence properties of the Si-LDS composite are stable under blue LED irradiation, and the fluorescence emission intensity of the film remains at $100 \%$ of its original value after the first 24 hours of irradiation. The Si-SRB composite, where the SRB molecules are not 
protected by the LDH matrix, exhibits a decrease in its luminescence features. After 2600 minutes, the fluorescence intensity had decreased by approximatively $40 \%$.

Thus, the added value of the LDH host matrix is well demonstrated, as is the stability of the Si-LDS composite film under thermal (up to $80^{\circ} \mathrm{C}$ ) and photonic stresses.
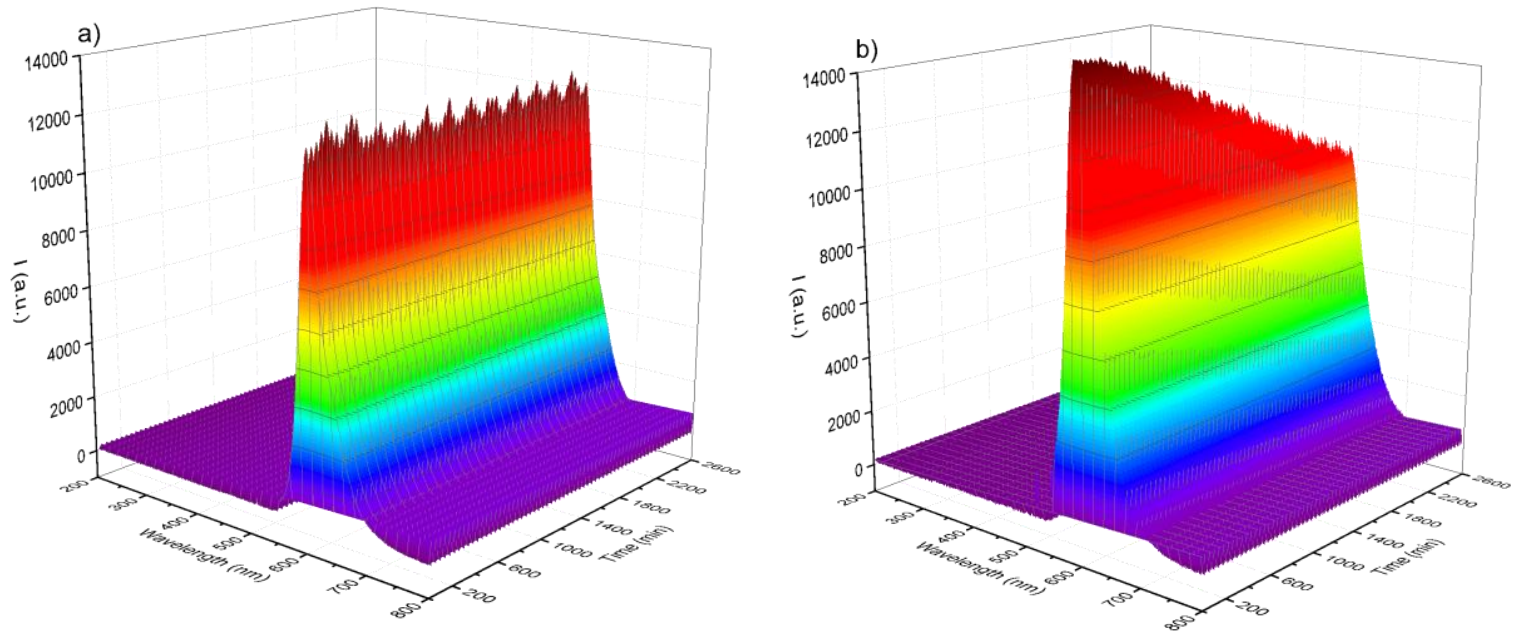

Figure 12. Variations in emission spectra plotted as a function of the irradiation time for both composites Si-LDS (a) and Si-SRB (b). The «sawtooth »-like shape is explained by the variation in blue LED power over time.

\section{Conclusions}

This study underlines the ability of a LDH host structure to co-incorporate concomitantly two organic molecules, DS and SRB. DS acts as a spacer, increasing the interlamellar space of the LDH sheets, thus propping apart the 2D-structure as well as rendering the LDH intergalleries organophilic. Moreover, the DS molecules are found to supply a dilution effect for SRB molecules, since the large quantity of DS forces the SRB molecules to be distant from each other, limiting their guest-guest interaction and their potential optical quenching. Experimentally, after precise screening, an optimized SRB input of $x=$ 0.002 is found to provide the hybrid LDH-DS-SRB with a suitable red emission under blue excitation.

The role of LDH in the production of the composite luminescent and homogeneous films is highlighted here. LDH platelets facilitate the dispersion of the SRB molecules in the silicone, while the SRB molecules tend to aggregate in the absence of LDH. Interestingly, Si-LDS films provide better photometric performances (CRI $=89.4$ and $\mathrm{Tc}=2700 \mathrm{~K}$ ) for white LED by enriching the emission spectra in combination with a YAG:Ce ${ }^{3+}$ composite film and a blueemitting LED. The composite films were found to be stable under photonic and thermal stresses applied to the LED device (100\% of the initial luminescence after one day of exposure), underlining clearly the stabilisation effect supplied by the LDH host matrix.

Finally, these findings open new perspectives for further improving LED technology. The stability of the fabricated composite films should now be investigated in depth to address the real potential of SRB molecules relying on an organomodified LDH toolbox.

\section{Conflicts of interest}

There are no conflicts to declare.

\section{Acknowledgements}

This work was supported by CPER DEFI MMASYF through its 2016 «MetaProfile» project. The authors would therefore like to thank the European Union, within the framework of the European Regional Development Fund (ERDF), and the Région Auvergne Rhône-Alpes, which co-funded this project.

\section{References}


1. G. B. Nair, H. C. Swart and S. J. Dhoble, Progress in Materials Science, 2020, 109, 100622.

2. Z. Xia, Z. Xu, M. Chen and Q. Liu, Dalton transactions, 2016, 45, 11214-11232.

3. E. Alonso, A. M. Sherman, T. J. Wallington, M. P. Everson, F. R. Field, R. Roth and R. E. Kirchain, Environmental Science \& Technology, 2012, 46, 3406-3414.

4. M. Humphries, Rare Earth Elements: The Global Supply Chain Congressional research service, 2013.

5. T. Hasegawa, Y. Kondo, Y. Koizumi, T. Sugiyama, A. Takeda, S. Ito and F. Hamada, Bioorganic \& Medicinal Chemistry, 2009, 17, 6015-6019.

6. L. V. Johnson, M. L. Walsh and L. B. Chen, Proceedings of the National Academy of Sciences, $1980,77,990$.

7. Z. Sun, L. Jin, W. Shi, M. Wei, D. G. Evans and X. Duan, Langmuir, 2011, 27, 7113-7120.

8. G. Lamouche, P. Lavallard and T. Gacoin, Physical Review A, 1999, 59, 4668-4674.

9. N. Sultana, Journal of Dispersion Science and Technology, 2018, 39, 92-99.

10. P. Legentil, F. Leroux, S. Therias, R. Mahiou and G. Chadeyron, Journal of Luminescence, 2019, 215, 116634.

11. D. Yan, J. Lu, M. Wei, D. G Evans and X. Duan, Sulforhodamine B Intercalated Layered Double Hydroxide Thin Film with Polarized Photoluminescence, 2009.

12. M. Ogawa and K. Kuroda, Chemical Reviews, 1995, 95, 399-438.

13. Q. Yin, D. Li, J. Zhang, Y. Zhao, C. Wang and J. Han, Applied Surface Science, 2019, 487, 1-8.

14. M. J. Wu, J. Z. Wu, J. Zhang, H. Chen, J. Z. Zhou, G. R. Qian, Z. P. Xu, Z. Du and Q. L. Rao, Catalysis Science \& Technology, 2018, 8, 1207-1228.

15. L. Yan, Y. Wang, J. Li, S. Kalytchuk, A. S. Susha, S. V. Kershaw, F. Yan, A. L. Rogach and X. Chen, Journal of Materials Chemistry C, 2014, 2, 4490.

16. E. Conterosito, I. Benesperi, V. Toson, D. Saccone, N. Barbero, L. Palin, C. Barolo, V. Gianotti and M. Milanesio, ChemSUSchem, 2016, 9, 1279-1289.

17. S. P. Lonkar, B. Kutlu, A. Leuteritz and G. Heinrich, Applied Clay Science, 2013, 71, 8-14.

18. H. Chen, Y. Wang, J. Lin, M. Shuai, H. Zhu, W. Zhang and Q. Ling, Journal of the American Ceramic Society, 2019, 102, 2718-2726.

19. H. Chen and W.-G. Zhang, Journal of the American Ceramic Society, 2010, 93, 2305-2310.

20. J. S. Bendall, M. Paderi, F. Ghigliotti, N. Li Pira, V. Lambertini, V. Lesnyak, N. Gaponik, G. Visimberga, A. Eychmüller, C. M. S. Torres, M. E. Welland, C. Gieck and L. Marchese, Advanced Functional Materials, 2010, 20, 3298-3302.

21. S. Cho, S. Jung, S. Jeong, J. Bang, J. Park, Y. Park and S. Kim, Langmuir, 2013, 29, 441-447.

22. W. Liu, S. Xu, S. Guan, R. Liang, M. Wei, D. G. Evans and X. Duan, Advanced materials, 2018, 30, 1704376.

23. V. Paolucci, S. L. Mejlsøe, M. Ficker, T. Vosch and J. B. Christensen, The Journal of Physical Chemistry B, 2016, 120, 9576-9580.

24. B.-J. Kim and K.-S. Kang, Materials Chemistry and Physics, 2014, 148, 964-967.

25. L. F. Vieira Ferreira, M. J. Lemos, M. J. Reis and A. M. Botelho do Rego, Langmuir, 2000, 16, 5673-5680.

26. V. Thangaraj, J. Bussiere, J.-M. Janot, M. Bechelany, M. Jaber, S. Subramanian, P. Miele and S. Balme, European Journal of Inorganic Chemistry, 2016, 2016, 2125-2130.

27. M. Ogawa, K. Saito and M. Sohmiya, Dalton transactions, 2014, 43, 10340-10354.

28. J. Bauer, P. Behrens, M. Speckbacher and H. Langhals, Advanced Functional Materials, 2003, 13, 241-248.

29. A. Clearfield, M. Kieke, J. Kwan, J. L. Colon and R.-C. Wang, Journal of inclusion phenomena and molecular recognition in chemistry, 1991, 11, 361-378.

30. S. De and R. Kundu, Journal of Photochemistry and Photobiology A: Chemistry, 2011, 223, 7181.

31. S. Das, A. P. Chattopadhyay and S. De, Journal of Photochemistry and Photobiology A: Chemistry, 2008, 197, 402-414.

32. K. Ray and H. Nakahara, The Journal of Physical Chemistry B, 2002, 106, 92-100. 
33. M. Wittmann and A. Penzkofer, Chemical Physics, 1993, 172, 339-348.

34. S. Srisantitham, M. Sukwattanasinitt and S. Unarunotai, Colloids and Surfaces A: Physicochemical and Engineering Aspects, 2018, 550, 123-131.

35. S. Daly, A. Kulesza, G. Knight, L. MacAleese, R. Antoine and P. Dugourd, The Journal of Physical Chemistry A, 2015, 119, 5634-5641.

36. X. Tang, Y. Liu and S. Li, RSC Advances, 2016, 6, 80501-80510.

37. J. L. Nyalosaso, R. Boonsin, P. Vialat, D. Boyer, G. Chadeyron, R. Mahiou and F. Leroux, Beilstein Journal of Nanotechnology, 2019, 10, 760-770.

38. G. S. Ranganath, Resonance, 2008, 13, 115-133. 